\title{
Functional PET Scan in Four Patients with Higher Order Neglect-Like Cognitive Dysfunction Associated to Chiasm Related Pathology
}

\author{
Hans Callø Fledelius ${ }^{1 *}$, Kirsten Korsholm², Ian Law ${ }^{2}$ \\ ${ }^{1}$ Copenhagen University Eye Clinics, Rigshospitalet and Glostrup Hospital, Capital Region, Denmark \\ ${ }^{2}$ Department of Clinical Physiology, Nuclear Medicine and PET, Rigshospitalet, Capital Region, Denmark \\ Email: ${ }^{*}$ hcfled@mail.dk
}

Received 9 February 2015; accepted 20 April 2015; published 21 April 2015

Copyright (C) 2015 by authors and Scientific Research Publishing Inc.

This work is licensed under the Creative Commons Attribution International License (CC BY).

http://creativecommons.org/licenses/by/4.0/

(c) (i) Open Access

\section{Abstract}

Cognitive disturbances with neglect-like features have been reported occasionally in patients with chiasmal disorders, so far however with no obvious substrate by conventional brain imaging. Thus, there were no right hemisphere lesions that could explain the lateralised visual inattention as observed in particular during monocular visual acuity testing. On this background, we further examined four adult patients who consented to functional ${ }^{18} \mathrm{~F}$-fluoro-deoxyglucose (FDG) positron emission tomography (PET) scan. In three there were no significant findings. The fourth patient, a 26-year-old male with cognitive defects after surgery for craniopharyngioma, will be discussed in more detail. His PET scan demonstrated a widespread reduction of regional metabolic activity in left hemisphere primary visual cortex and higher order visual areas, despite absence of explanatory pathological signal changes on MRI. As present in only one out of four patients, however, the findings do not allow specific pathogenetic mechanisms to be suggested, nor generally to substantiate involvement of higher cerebral circuits. Obviously, even developed imaging has its limits, and in the very theory the visual dysfunctions observed might still depend on higher brain centres' faulty adaptation to loss of pre-geniculate visual information.

\section{Keywords}

Chiasmal Lesions, Visual Field Defects, Neglect-Like Behaviour, Cognitive Disturbances, Functional PET Scan (FDG) of Brain

\footnotetext{
${ }^{*}$ Corresponding author.
}

How to cite this paper: Fledelius, H.C., Korsholm, K. and Law, I. (2015) Functional PET Scan in Four Patients with Higher Order Neglect-Like Cognitive Dysfunction Associated to Chiasm Related Pathology. Open Journal of Ophthalmology, 5, 54-60. http://dx.doi.org/10.4236/ojoph.2015.52009 


\section{Introduction}

Reading can be disturbed out of proportion to visual acuity findings in patients with lesions of the chiasm, and some patients cannot mobilize the search saccades as usually triggered during monocular visual acuity testing. Accordingly they show neglect-like visual inattention to the temporal part of the visual chart [1]-[4]. Various neuro-psychological tests have confirmed the occurrence of cognitive disturbances [5], but patho-physiologic mechanisms are not clear. MRI scan had given no clue in such cases, and after written and oral information and patients' consent ${ }^{18} \mathrm{~F}$-fluoro-deoxyglucose (FDG) PET scanning of the brain was performed, in a search for deviating metabolic patterns. Four adult patients were studied, however with significant deviation from normal in only one, a young male aged 26 who suffered from sequels after surgery for an extensive craniopharyngioma.

To stress the clinical similarities, all four case stories are given below, though with more detail regarding the young male patient who has shown positive findings on the functional PET scan (Case No. 4).

In respect of the tenets of the Helsinki Declaration, all had given informed consent to the study.

\section{Case Reports}

\subsection{Case 1}

A 53-year-old female laboratory technician with well controlled diabetes type 2 had visual disturbances of the left eye over 2 months, when first seen. Best corrected visual acuities (BCVA) were 1.0 and 0.4, and the left eye further had dyschromatopsia (Ishiharas plates). Initial tangent screen examinations (white 5/1000) indicated normal visual fields, but Octopus static perimetry subsequently suggested relative bitemporal loss of sensitivity, and CT scan demonstrated a $3 \times 3 \times 3.5 \mathrm{~cm}$ suprasellar tumour. Visual complaints worsened after extirpation of a pituitary adenoma, visual acuities fell to 0.8 and 0.16 , and both eyes showed neglect-like temporal blocking on the visual chart when tested monocularly. She further presented temporal hemianopia for all isoptres, and the absolute losses of the left eye even crossed the mid-vertical. Binocular reading was hampered due to "loosing words”, and cognition in space presented misinterpretations. Optic disc atrophy developed.

By neuro-psychological testing her left eye showed substantial losses by Gothenburg test, BIT star test, and chimeric faces. The right eye only had slight losses, but cofunction was poor when binocular.

Only slight improvement over 11 years follow-up. A functional FDG PET scan was added in 2012, however without indicative findings.

She is on a pension.

\subsection{Case 2}

A 39-year-old locksmith with headache and visual loss over a month, BCVA at admission 0.1 and 1.0. Tangent screen showed inferior temporal defect of right eye, left eye normal. His orientation and navigation in space became severely impaired after extirpation of a $2.5 \times 2.5 \times 2.5 \mathrm{~cm}$ pituitary tumour, despite a maintained BCVA of the left eye of 1.0. Visual testing, however, demanded extra time, and the right eye had not improved. Massive bitemporal visual field losses were demonstrated, to midline of the better eye and above midline for the right eye. Both optic discs were pale, and a 6 pd horisontal diplopia could not be relieved by prisms. By monocular visual acuity testing the right eye kept the pattern of catching only the nasal column on the chart, whereas eventually the left eye could compensate for the initial neglect-like temporal ignorance and, letter by letter, deliver the full line. Reading a text has remained slow and fragmented.

At most neuropsychological tests, his right eye could not cope with the demands. The Gothenburg test, BIT star test, chimeric faces and line division were passed close to normal for the left eye, provided he was allowed to choose a skew gaze direction.

Only slight functional improvement over 10 years follow up, when he had functional FDG PET scan performed, in 2013, without explanatory findings.

He is on a pension.

\subsection{Case 3}

A 42-year-old female office clerk was referred for bilateral loss of visual acuity and field. At admission right eye BCVA was 0.25 and left eye 0.7. Colour sense was normal, but reading a text was slow. Surgery for cranio- 
pharyngioma appeared uneventful, but four days later malaise and loss of vision occurred. Monocular testing of vision was not feasible, and binocularly she could hardly catch the visual chart at a distance of 1 meter. The right eye however soon improved, to 0.9 BCVA, though with full ignorance of the temporally located test symbols on the chart. The left eye had dropped to visual acuity 0.03 , but conceived only on and off, nasally on the chart. A massive bitemporal hemianopia tended to incorporate the vertical midline in the defect. Poor reading ability (easier vertically!) and spatial disorientation were also noted. Optic discs pale, partly atrophic.

Neuropsychological testing: Only moderate drop out by all tests, provided lots of time allowed; reading slow with loss of words. In the binocular situation trouble regarding eye-hand coordination.

Still severely incapacitated in everyday functions, over 10 years, and no explanatory findings at the FDG PET scan performed in 2012.

She is on a pension.

\subsection{Case 4}

A 26-year-old otherwise healthy male IT technician had a large parasellar tumour diagnosed on MRI scan of the brain. The exam was prompted by general loss of concentration, headache, and fainting episodes over 2 - 3 weeks, and ophthalmic evaluation was not carried out prior to the first neurosurgical approach, in December 2009. This had the character of an emergency decompression, a large cystic craniopharyngioma being partially removed transnasally. Immediately after surgery he had best corrected visual acuities of 0.6 right eye and 0.4 left eye, and bitemporal hemianopia was recorded.

Transcranial extirpation of residual tumour was performed one year later, based mainly on poor capacity in his job and a considerable weight gain. A 9 mm suprasellar cyst formation on MRI led to stereotactic radiotherapy (July 2012).

Reading a text had become increasingly difficult, sometimes also cognition in space, and he is currently followed in the University Clinic for Brain Damage. He is under hormone substitution for pituitary insufficiency and has had a single episode with convulsions.

His post-radiation ophthalmic state has been stable. Each eye can yield a slow 0.4 decimal visual acuity, however with an obvious nasal preference on the chart, manifesting as visual ignorance and "blocking” of the search saccades to the temporal side. Using both eyes, he can compensate for the lateral attention deficit and also read a medium size text (N8), though slowly and without natural rhythm. There is a left eye relative afferent pupil defect.

Both eyes had complete loss of colour sense, whereas contrast sensitivity was reduced only slightly (PelliRobson). Kinetic Goldmann and static Octopus perimetry both indicated bitemporal hemianopia (Figure 1). Pursuit eye movements appeared normal and alternate cover test indicated a slight exophoria. A hemifield slide disturbance could not be demonstrated by Schober's test (red-green dissociation), where the left eye image was ignored or suppressed most of the time. Except slight pallor of both optic discs fundus examination was normal, and MRI scan in 2013 has given no indication of tumour recurrence.

The FDG PET scan was performed in 2013. In addition to visual analysis, a quantitative 3-dimensional stereotactic surface projection analysis (Scenium (v 3.0), Siemens) was employed that allowed direct visualization of the extent and the topography of FDG uptake abnormalities. In addition, the PET images were co-registered to T1 and T2 weighted cerebral MRI scanning performed 7 months before, for identification of structural pathology and identification of anatomical structures. Significant metabolic reduction compared to a database of healthy subjects were found in the orbitofrontal cortex corresponding to treatment related damage after surgery, but also in multiple left posterior cortical areas known from extensive clinical studies and brain mapping experiments to subserve functions in the computation of visual input [6] [7]: the medial occipital region in the posterior part of the calcarine fissure, the inferior parietal lobe, and the inferior temporal lobe, starting from the occipito-temporal junction and extending into the temporal pole (Figure 2 and Figure 3). Corresponding to these areas MRI had shown no explanatory pathological signal changes.

\section{Discussion}

As for the clinical features, emphasis is on the neglect-like attentional deficits as evident in all four patients when testing the visual acuity for each eye. Clinically, they all belong to the heavy tail of the chiasmal spectrum, here factually to include cognitive dysfunction. The patient thus knows there is a full line of optotypes, but 

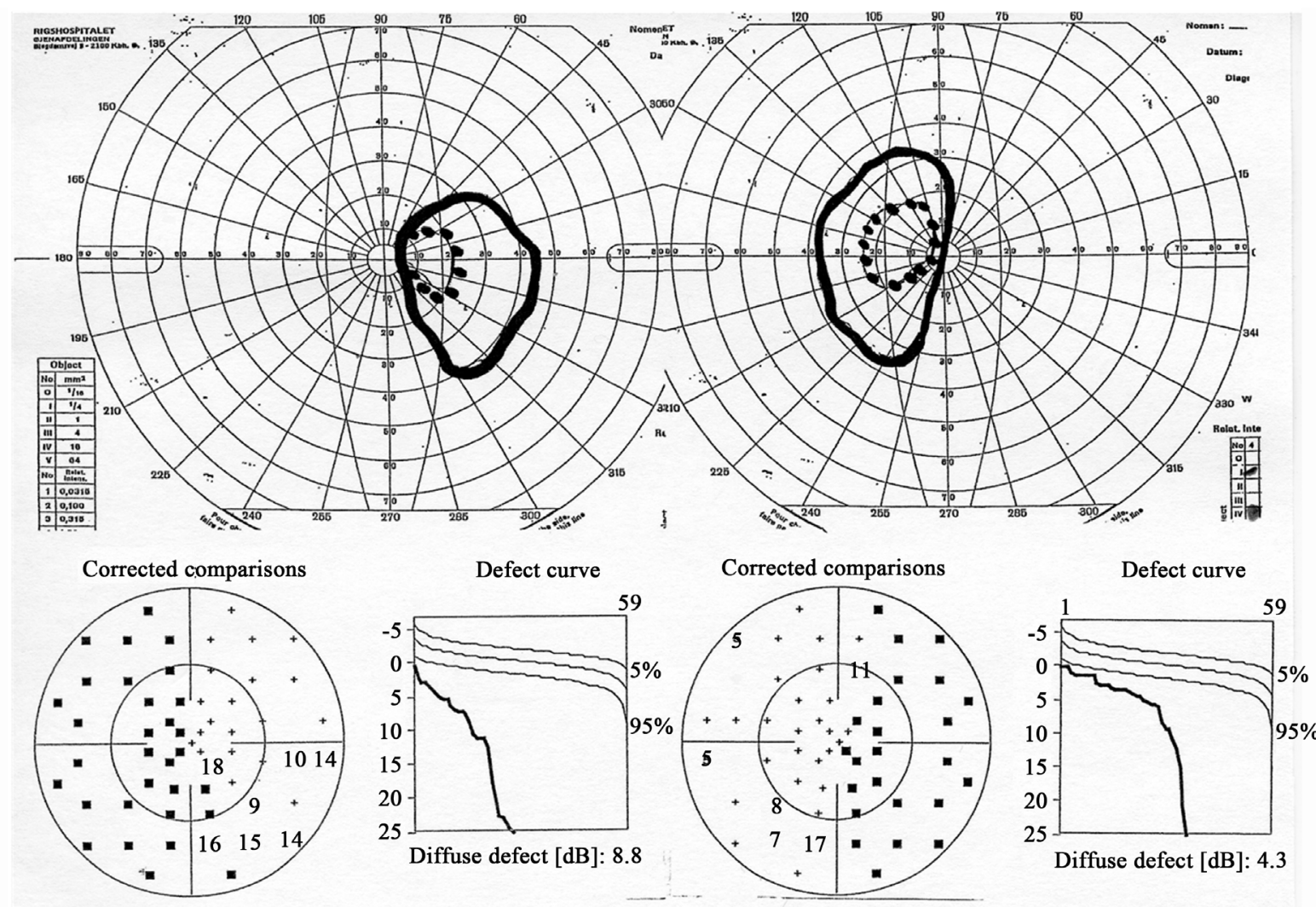

Figure 1. On top, kinetic Goldmann perimetry, white objects IV, 4 and I, 4, showing the borders of preserved nasal fields, apparently leaving a vertical midline bar of non-attention. Bottom: static octopus G standard programme, central $30^{\circ}$ field, black squares indicating bitemporal absolute scotoma test points; cumulated decibel loss of sensitivity is further demonstrated.

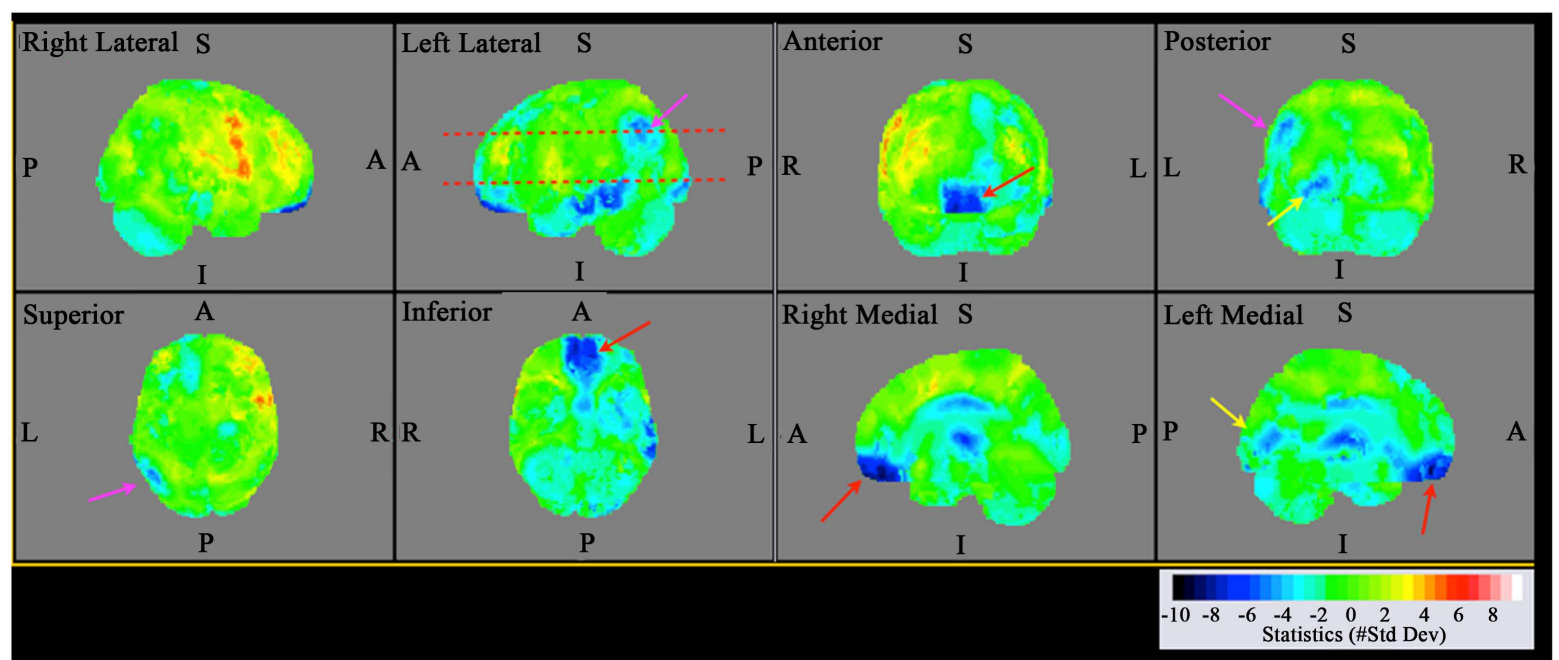

Figure 2. Statistical surface projection comparing the patient's regional metabolic activity to a database of healthy age matched subjects showing right and left lateral, and anterior and posterior projections (top), and superior, inferior, and right and left medial projections (bottom). There are significant reductions in the orbitofrontal cortex (red arrow) corresponding to the neurosurgical route of entrance, and to remote locations in left hemisphere visual areas, such as the primary visual cortex (yellow arrow), inferior parietal lobe (pink arrow), and inferior temporal lobe. The scale refers to standard deviations. A Z-score of $<3$ is considered significant. The two dotted lines denote approximate levels of the slices in Figure 3. 


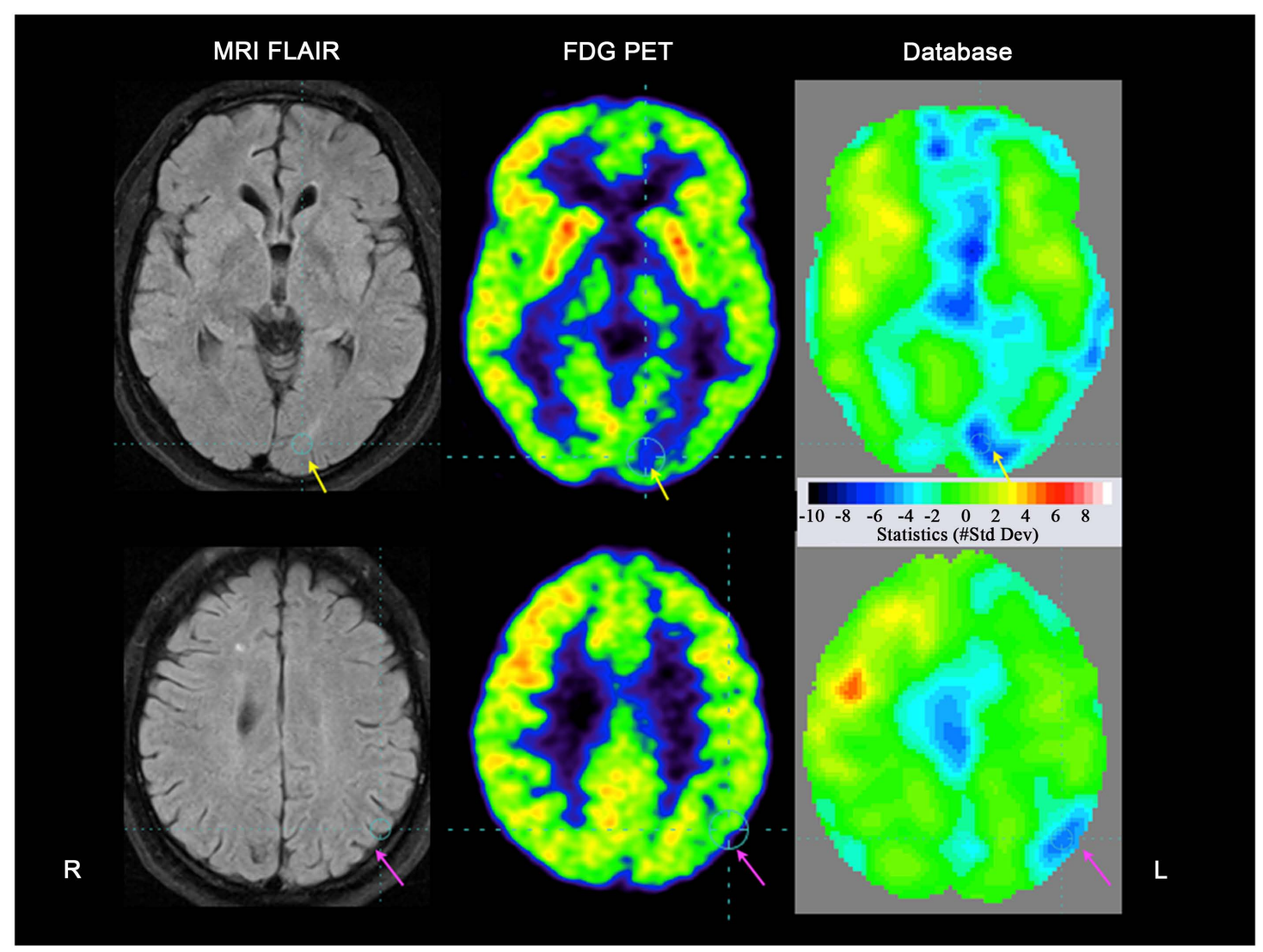

Figure 3. Two trans-axial brain imaging slices corresponding to the levels denoted in Figure 2 showing MRI FLAIR, FDG PET, and a statistical comparison of FDG PET to a database of healthy subjects. Significant metabolic reductions are seen in left hemisphere in the primary visual area close to the calcarine fissure (yellow arrow), and in the inferior parietal lobe (pink arrow). There is an impression of diffusely reduced metabolism in the whole left hemisphere, which increases the right prefrontal cortex relatively. A Z-score of $<3(\mathrm{p}<0.001)$ is considered significant.

appears functionally blocked to what is located on the temporal side of the chart and reads only what is nasal [1] [3] [4]. Obviously, the usual search saccades cannot be triggered. The patients further presented loss of former abilities also when binocular, for instance when confronted with a simple text, in print or on screen [5].

The left eye Goldmann perimetry isoptres shown in Figure 1 illustrate an apparent paradox, as evident also in the worse eye of the other patients under study: fixation is maintained and centred in the bottom of the Goldmann globe despite the apparent position of the fixation mark in the recorded scotoma zone. Goldmann recordings might further suggest a blind central bar along the mid-vertical in binocular visual space, but this is refuted by hand movements (testing by bimanual confrontation) and likewise by targeted small object (white 5/1000) binocular tangent screen examination. This seems in accord with many chiasmal patients' lack of noticing visual field trouble, in part obviously by virtue of the two mainly undisturbed nasal half-fields. An element of neglect may further contribute to ignorance, and the apparent absence of complaints may explain clinicians' occasional omission of early visual field testing. However, the striking performance at customary unilateral visual acuity testing should alert the medical staff and trigger the essential evaluation of the visual field. The specific neglect-like behaviour of nasal reading/temporal blocking is only sporadically emphasised in textbooks [1] [2]. In earlier clinical series we gave lengthy consideration to the clinical variety. It may range from solid evidence to discreet hints only, it can be uni- or bilateral, and complaints may be minimal [3]-[5].

The distribution of relative regional cerebral glucose metabolic rate (rCMRglc) is recognized as an indirect imaging biomarker of neural activity, as assessed here by FDG PET scanning [6] [7]) The expectations were that the PET scanning of the brain might suggest compensatory functional pathways in the brain of relevance for the deviant visual behaviour under study, possibly to indicate dysfunction of higher order visual areas. For instance, this could be through a coupled functional deactivation or disconnection syndrome known as diaschisis, either 
by the loss of afferent neural input via connecting white matter tracts from the primary cortex (V1), or through the lateral geniculate body [8]-[10]. Diaschisis is a frequent clinical functional neuroimaging finding in other contexts, e.g. crossed cerebellar diaschisis in patients with frontal lobe damage.

Attentional neglect may include disordered saccades. Most often it is associated to lesions in the right inferior parietal lobe, however with a definite role also for the frontal lobe, as based on frontoparietal and other network associations [11]-[16]. Splenial interhemispheric disconnection has further been reported, with the authors' suggestion that deprivation of sensory inputs to intact parietal and frontal areas can also yield spatial neglect limited to specific sensory modalities or sectors of space [17]. Imaging in our feature case (patient No. 4) presented extensive frontal lobe defects, as direct sequels to the open brain surgery performed. Possibly this was a co-factor underlying his attentional deficits [18], but similar lesions of a frontal location were not disclosed in the other patients of the present series.

Comparing clinical features in general, another item should be mentioned: Typical neglect is on a proven lesional basis, with emphasis on macroscopic gray and white matter pathology, usually in the right hemisphere and presenting binocular visuo-spatial neglect in the left hemifield. In contrast, the characteristic single eye neglect-like features in chiasmal patients may lateralise to either side, and damage to the optic pathways usually appears the main or only lesion to brain tissue. Thus there is no obvious structural link to the pathology that typically underlies the attentional deficits known as classical visual neglect.

\section{Conclusions}

In summary, chiasmal patients' occasional neglect-like behaviour at single eye vision testing, sometimes combined with cognitive disturbances, has been recognized for decades. Neuro-psychological evaluations and MRI scans of the brain, however, have not disclosed obvious pathophysiological mechanisms. This motivated the present study, where functional PET scan presented evidence of suprageniculate functional deficits in cortical areas known to play a role in primary visual and visuo-spatial analysis, though only in one of the four chiasmal patients actually under study. Accordingly, more specific generalisations are not allowed regarding abnormal brain processing as possibly associated to pre-geniculate visual pathway disorders. In the theory, however, such pathology might have curtailed information to supragenicular networks usually considered of importance for cognition in free space [12]-[16].

Our second aim with the presentation was to direct the attention of ophthalmic colleagues when confronted with reading trouble out of proportion to visual acuity findings in young patients—and/or the striking monocular lateralising behaviour on a visual chart—as potential early markers of chiasmal pathology.

\section{References}

[1] Glaser, J.S. (1990) Neuro-Ophthalmology. 2nd Edition, Lippincott, Philadelphia, 12, 172.

[2] Miller, N.R. and Newman, N.J. (1999) Topical Diagnosis of Chiasmal and Retriochiasmal Lesions. In: Walsh, F.B. and Hoyt, W.F., Eds., Clinical Neurophthalmology, 5th Edition, Lippincott, Philadelphia, 326-335.

[3] Fledelius, H.C. (2004) Chiasmal Pathology Causing Inability to Access Information in the Temporal Visual Field. Neuro-Ophthalmology, 28, 77-85. http://dx.doi.org/10.1076/noph.28.2.77.23740

[4] Fledelius, H.C. (2009) Temporal Visual Field Defects Are Associated with Monocular Inattention in Chiasmal Pathology. Acta Ophthalmologica, 87, 769-775. http://dx.doi.org/10.1111/j.1755-3768.2008.01328.x

[5] Fledelius, H.C. (2014) Neuropsychological Testing in Chiasmal Patients Exhibiting Inattention in the Temporal Visual Space during Monocular Testing. Neuro-Ophthalmology, Accepted for Publication.

[6] Gerlach, G., Law, I.A., Gade, A. and Paulson, O.B. (1999) Perceptual Differentiation and Category Effects in Normal Object Recognition: A PET Study. Brain, 122, 2159-2170. http://dx.doi.org/10.1093/brain/122.11.2159

[7] Nobre, A.C., Sebestyen, G.N., Gitelman, D.R., Mesulam, M.M., Frankowiak, R.S. and Frith, C.D. (1997) Functional Localisation of the System for Visuospatial Attention Using Positron Emission Tomography. Brain, 120, 515-533. http://dx.doi.org/10.1093/brain/120.3.515

[8] Von Monakow, C. (1914) Die Lokalisation in Grosshirn unter der Abbau der Funktion durch kortikale Herde. JF Bergmann, Wiesbaden.

[9] Finger, S., Koehler, J. and Jagella, C. (2004) The Monakow Concept of Diaschisis. Origin and Perspectives. Archives of Neurology, 61, 283-288. http://dx.doi.org/10.1001/archneur.61.2.283

[10] Baron, J.C., Bousser, M.G., Comar, D., Sousaline, F. and Castaigne, P. (1981) Non-Invasive Tomographic Study of 
Cerebral Bloodflow and Oxygen Metabolism in Vivo. Potentials, Limitations, and Clinical Applications in Cerebral Ischemic Disorders. European Neurology, 20, 273-284. http://dx.doi.org/10.1159/000115247

[11] Ringman, J.M., Saver, J.L., Woolson, R.E., Clarke, S.W.R. and Adams, H.P. (2004) Frequency, Risk Factors, Anatomy, and Course of Unilateral Neglect in an Acute Stroke Cohort. Neurology, 63, 468-474.

http://dx.doi.org/10.1212/01.WNL.0000133011.10689.CE

[12] Swan, L. (2001) Unilateral Spatial Neglect. Physical Therapy, 81, 1572-1580.

[13] Bartolomeo, P., Thiebaut de Schotten, M. and Chica, A.B. (2012) Brain Networks of Visuospatial Attention and Their Disruption in Visual Neglect. Frontiers in Human Neuroscience, 6, Article 110. http://dx.doi.org/10.3389/fnhum.2012.00110

[14] Ptak, R. and Fellrath, J. (2013) Spatial Neglect and the Neural Coding of Attentional Priority. Neuroscience and Biobehavioral Reviews, 37, 705-722. http://dx.doi.org/10.1016/j.neubiorev.2013.01.026

[15] Balslev, D., Odoj, B. and Karnath, H.O. (2013) Role of Somatosensory Cortex in Visuospatial Attention. Journal of Neuroscience, 33, 1311-1318. http://dx.doi.org/10.1523/JNEUROSCI.1112-13.2013

[16] Mesulam, M.M. (1981) A Cortical Network for Directed Attention and Unilateral Neglect. Annals of Neurology, 10, 309-325. http://dx.doi.org/10.1002/ana.410100402

[17] Tomaiulolo, F., Voci, L., Bresci, M., Cozza, A., Posterano, F., et al. (2010) Selective Visual Neglect in Right Brain Damaged Patients with Splenial Interhemispheric Disconnection. Experimental Brain Research, 206, 209-217. http://dx.doi.org/10.1007/s00221-010-2230-6

[18] Stone, J., Renolds, M.R. and Leuthardt, E.C. (2011) Transient Hemispatial Neglect after Surgical Resection of a Right Frontal Lobe Mass. World Neurosurgery, 76, 361. http://dx.doi.org/10.1016/j.wneu.2010.03.018 